\title{
Can an Emergency Surgery Scheduling Software Improve Residents' Time Management and Quality of Life?
}

\author{
James Lee ${ }^{1}$ | Ahmed Aoude ${ }^{1}$ | Becher Alhalabi ${ }^{1}$ | Ayden Watt ${ }^{2}$ | \\ Lucie Lessard $^{1}$
}

${ }^{1}$ Department of Surgery, McGill University Health Centre, Montreal, QC, Canada

${ }^{2}$ Department of Experimental Surgery, McGill University

Correspondence

James Lee

Email: james.lee@muhc.mcgill.ca

Publication Date

October 18, 2021

MJM 2022 (20) 15

https://doi.org/10.26443/mjm.v20i1.893

\section{McGill}

\section{Journal of Medicine}

www.mjmmed.com

\section{(c) (i) (2) (2)}

This work is licensed under a Creative Commons BY-NC-SA 4.0 International License.

\section{A B S TRACT}

Background: Operating room efficiency is invaluable. Particularly in public health systems, where resources are limited and patient loads are high, efficient systems underpin the continued delivery of high quality care. In addition to impacting patients, the implementation of efficient healthcare tools has the potential to improve staff quality of life. In the face of growing surgical resident attrition and healthcare worker burnout, developments in standard practice, such as the implementation of the 80hour work week, are necessary to improve quality of life.

Materials and Methods: A new online scheduling software (ORNET.CA) was created, installed, and piloted in a Level I Trauma Center after instructing users (physicians and nurses) on its use. A 20-item survey was then distributed to all users to assess the effect implementation of the software had on their quality of life.

Results: ORnet was shown to improve communication between hospital staff and physicians, reduce workflow interruptions, and improve the quality of the working environment. The survey showed that $60 \%$ of residents and $50 \%$ of attending staff believed that ORNET.CA improved their quality of life.

Conclusions: We present data from a novel emergency operating room scheduling system that allowed surgical residents and attending physicians to better plan their on-call shifts. Staff (resident and physician) reported survey results suggest that implementation of this system resulted in an improved quality of life and a decrease in stress and anxiety levels.

\section{KEYWORDS}

Quality of life, Operating room scheduling, Surgical residency, Operating room management 


\section{1 | INTRODUCTION}

The regular day to day activities of residents in surgical specialities are very demanding. Studies examining all operating room staff show that residents have more work burden, longer work hours, more physical work, and high levels of stress. (1-5) In one study, the majority of surgical residents noted that they experience workrelated stress that is moderate to severe. (5) These residents also reported to be drowsy during the daytime and that the stress affected their overall well-being. (5)

The higher stress situation that surgical residents go through partly explains higher burnout rates among surgical residents. (2) Multiple studies have looked at the reasons for this, and in one study, future lifestyle, sleep deprivation, and work hours were the main reasons residents decided to quit residency. (1) The higher attrition rates amongst surgical residents also affects work quality, personal and family problems and in turn affects patient care. (5) Solutions attempting to decrease attrition rates in surgical programs have included: allowing for post call days, decreasing maximum number of work hours, and providing diverse support methods for residents throughout their residency. However, some studies report negative outcomes even after the implementation of these methods. $(6,7)$ In addition, little or no literature exists on methods to improve time management for residents, particularly during on-call duties.

Being a surgical resident in Canada differs from the United States because of less operating room (OR) resources in a Governmental Health Care system. (8) In Canada, emergency non-elective cases are more likely take place during evening and weekends since operating room resources are limited; non-elective surgery is typically limited to one operating room per institution in Canada and performed only once elective cases during the day have all been completed. $(9,10)$ Therefore, all surgical specialities struggle to get their emergency nonelective cases done while only one OR room is running. Rarely would two services be operating at the same time in two different rooms unless a life-or-death situation exists. Hence, cases being done later in the evening or night become more common. (9)
In this paper, we present a novel software implemented and used in a Level I Trauma Center intended to improve communication and allow residents to better predict the emergency room operating room schedule and in turn improve time management and quality of life.

\section{2 | METHODS}

A new online scheduling software ORNET.CA (Montreal, Canada) was created and installed in a Level I Trauma Center (test site). All nursing staff were trained for its use. Physicians were also sent an email with instructions on its use. The software was launched in October 2015. The software depicts the operating room (OR) schedule on weeknights or evenings (emergency non-elective OR time). OR nursing staff input the list of emergency nonelective cases for the day in real-time based on priority classification levels established by the health care center using it. On-call physicians then log in to the web based real-time scheduling software and view when their case would start and if the start time has been advanced or delayed due to other emergency cases being completed or booked. In a situation where an OR is delayed due to emergency, the physicians on-call are notified immediately via e-mail/text message notification or via the webbased scheduling software directly. Information about scheduled cases, start times, and equipment needed is visible to the health care professionals in real time.

A short online survey was emailed to all 133 users (from the departments of orthopaedic surgery, general surgery, and plastic surgery) in April 2018 to determine quality of life measures and effects of the software on its users. This survey consisted of 20-items and was designed in English (Appendix A) for surgical physicians working at the Montreal General Hospital (MGH). Respondents were given a 1-month time frame to answer the survey, which inquired about the usability of the software and its effects on time management and quality of life. A request to participate in this online survey was sent to all the surgical residents and attending surgery staff at the site electronically, including a link to 
anonymously complete the questionnaire. Descriptive statistics using Microsoft Excel 2019 were used to analyze the survey results. Responses were analyzed and grouped based on answer likelihood.

No patient information was present on the scheduling software to ensure patient confidentiality. Only procedure, expected surgical time, and surgical department was shown on the scheduling software. This allowed for communication of start times, surgical equipment requirements for each case, and allowed for real-time case flow management without compromising patient privacy.

\section{\begin{tabular}{l|l}
3 & RESULTS
\end{tabular}}

A total of 133 surveys were sent out, with 68 respondents representing a 51\% response rate. However, 20 responses were not completed and were excluded from our analysis. 30 surgical residents and 18 surgical staff (36.1\% of total surveys) fully completed the survey and their results were analyzed. While the $36.1 \%$ finalinclusion response rate is low, the authors judge that the survey was likely answered primarily by the physicians who used it most often. Senior residents were more likely to respond to the survey as they are on OR call more frequently than their junior colleagues. This population would thereby have the hand-on experience required to judge the effect of the software on quality of life, and return an accurate overview of the software's impact. $67 \%$ of included physicians responded that they use ORNET.CA very often or often (more than 6 times/month) during their on-call shifts, with all other physicians reporting occasional use of the software (35 times/month). $93 \%$ of residents used the software regularly to determine when a case was scheduled to start. In addition, 83\% of residents believed ORNET.CA improved communication and reduced the number of phone calls to the OR by at least $25-50 \%$ in comparison to prior to the software being implemented. Half the attending physicians also responded similarly.

\section{1 | Time Management}

When asked if ORNET.CA helped improve time management, $80 \%$ of residents (Figure 1.) and $44 \%$ of attending physicians agreed or strongly agreed. In addition, 68\% of residents (Figure 2.) and $39 \%$ of attending physicians agreed that ORNET.CA helped plan their evenings and weekends while on-call to engage in wellness activities, complete errands, and plan their study time more efficiently.

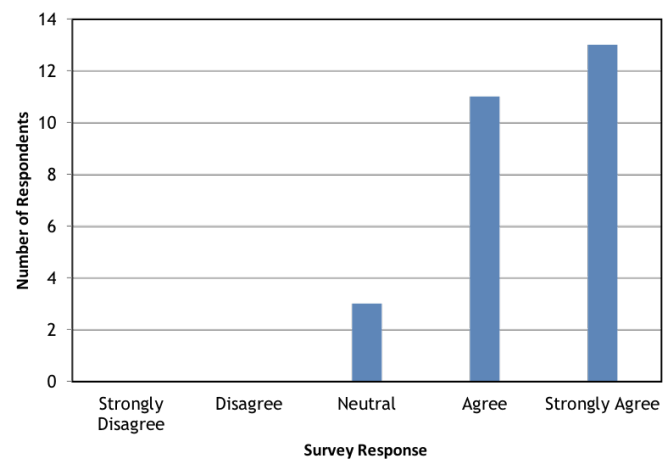

FIGURE 1 Resident Survey Results: ORnet is useful to help manage your time during your on-call shifts

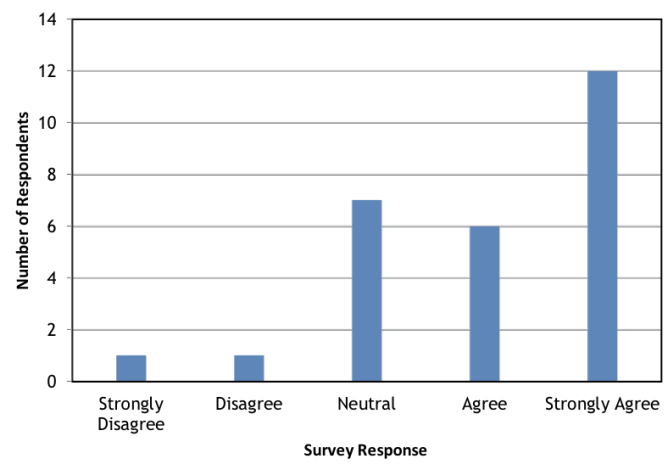

FIGURE 2 Resident Survey Results: ORnet has helped plan your evenings/weekend on-call to engage in wellness activities or errands 


\section{2 | Quality of Life}

When asked if ORNET.CA helps reduce anxiety and stress while on-call, $47 \%$ of residents agreed or strongly agreed (Figure 3.), whereas only $22 \%$ of attending staff agreed or strongly agreed. In addition, $60 \%$ of residents (Figure 4.) and 50\% of attending staff believed that ORNET.CA improved their quality of life.

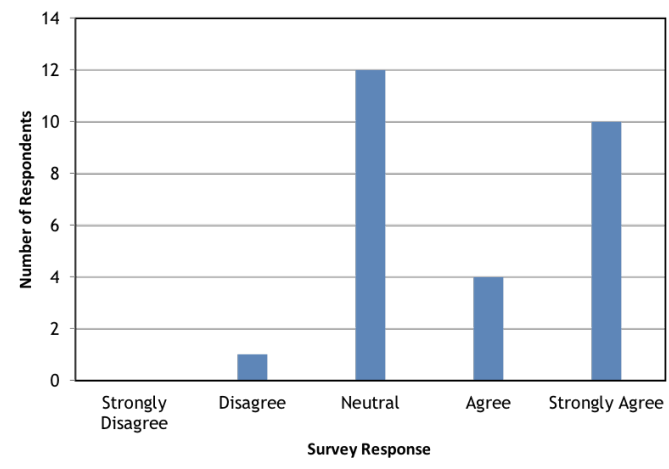

FIGURE 3 Resident Survey Results: ORnet has helped reduced stress/anxiety during on-call shifts

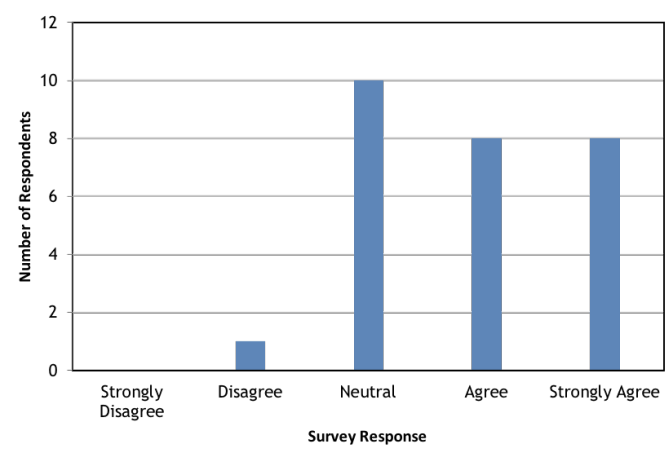

FIGURE 4 Resident Survey Results: ORnet has helped improve quality of life

\section{3 | Time Management Communication and Usefulness}

The majority of residents (67\%) and $39 \%$ of attending physicians believed that the software improves commu- nication between nurses and physicians in the OR. Additionally, $56 \%$ of attending physicians and $73 \%$ of residents believed that ORNET.CA is useful and should be continued to be used for emergency OR scheduling. Although recently adopted and launched as a pilot project at the MGH, the majority of users find ORNET.CA useful, improving communication and quality of life for those using it.

\section{4 | DISCUSSION}

This paper presents a new real-time scheduling software with visual depiction of cases on the emergency list used in a Level 1 Trauma Center in Canada and investigates its ability in improving communication between operating room staff and its ability to improve physician and resident quality of life. As demonstrated by the results of an online survey, this simple solution allowed for physicians and residents to better predict their on-call duties and in turn help them rest when possible or engage in wellness activities.

Surgical residents' well-being and attrition rates have become an important topic for discussion over the past few years. (1-7, 11) With high surgical resident attrition rates in the United States and Canada, research has focused on reasons for the high drop-off rates. The literature consistently showed that long working hours, (1) future career lifestyle, $(1,11)$ and sleep deprivation to be common reasons for residents to not complete their residency training. (1) With attrition rates as high as $17 \%$, (11) workload and environment have been questioned. Therefore, resident well-being has become important for all surgical programs. In this paper, we present data that shows that a simple scheduling software allowed residents to better plan their time while on-call, which contributed to improved quality of life. Our results also found that the software program was able to decrease stress levels and anxiety, which have been linked to resident burnout. (5)

Solutions to minimize surgical resident attrition have been investigated in the United states. $(6,7)$ Some studies have shown that strategies such as work hour restric- 
tion and post-call days have decreased residents' time in the hospital but has consequently negatively affected patient care and resident education. (6) Many studies demonstrate that resident stress is high and related to work hours, $(1,4)$ but limited studies have investigated the effect of stress related to unpredictable work hours and inability to schedule activities while on-call. Here we present a novel method for physicians, residents, and other hospital personnel to better predict their on-call shifts. This new software allows for residents to verify in real-time when their case will be expected to start and verify as frequently as they would like to in the matter of seconds rather than spending minutes to call the OR charge nurse. Therefore, they are able to better predict if they have time to have dinner, get some sleep, plan a basic life task such a grocery shopping, or schedule study time. We believe that taking away some of the unpredictability helps reduce their stress and improve quality of life as shown in the survey results.

Although the software used here was implemented for use by all OR personnel, the survey demonstrates that its effect in decreasing anxiety/stress levels and improving quality of life was more impactful for residents compared to attending staff. This is consistent with the literature that shows the likelihood of burnout due to stress is higher in residents than other OR personnel, including nurses. (3) This can be related to the fact that other operating room staff have fixed shift schedules while residents have more variability in their work hours and highly unpredictable on-call shifts. In addition, in most circumstances, residents are more frequently oncall than attending physicians. Hence, the use of the software presented here allows for better predictability and allows for stress reduction amongst residents as shown in the survey results.

One limitation of this study is that the results are taken from the experience of one center in Canada. (12) Other centers may report different perspectives on the use of the software, especially in the United States where more resources are available and emergency nonelective OR time is less scarce. (12) In addition, our survey results stem from a small number of surgical residents at the a single tertiary care center. Future stud- ies will look at the use of the software in more centers, including community centers, and determine if differences exist. (12)

\section{CONCLUSION}

Here we present data from a survey after the use of a novel emergency operating room schedule implemented in a level trauma center in Canada. The results show that a simple visual scheduling software allows surgical residents and attending physicians to better predict their on-call shifts. The results also show that a simple scheduling software accessible to all allows for improvement in quality of life and a decrease in stress and anxiety levels amongst residents. This in turn could potentially equate to a reduction in attrition rates among surgical residents.

\section{REFERENCES}

1. Gifford E, Galante J, Kaji AH, Nguyen V, Nelson MT, Sidwell RA, et al. Factors associated with general surgery residents' desire to leave residency programs: a multi-institutional study. JAMA Surg. 2014;149(9):948-53.

2. Hochberg MS, Berman RS, Kalet AL, Zabar SR, Gillespie C, Pachter HL. The stress of residency: recognizing the signs of depression and suicide in you and your fellow residents. Am J Surg. 2013;205(2):141-6.

3. Hyman SA, Michaels DR, Berry JM, Schildcrout JS, Mercaldo ND, Weinger MB. Risk of burnout in perioperative clinicians: a survey study and literature review. Anesthesiology. 2011;114(1):194204.

4. Lebensohn P, Dodds S, Benn R, Brooks AJ, Birch M, Cook P, et al. Resident wellness behaviors: relationship to stress, depression, and burnout. Fam Med. 2013;45(8):541-9.

5. Yoo PS, Tackett JJ, Maxfield MW, Fisher R, Huot SJ, Longo WE. Personal and Professional Well-Being of Surgical Residents in New England. J Am Coll Surg. 2017;224(6):1015-9.

6. Antiel RM, Reed DA, Van Arendonk KJ, Wightman SC, Hall DE, Porterfield JR, et al. Effects of duty hour restrictions on core competencies, education, quality of life, and burnout among general surgery interns. JAMA Surg. 2013;148(5):448-55.

7. Lindeman BM, Sacks BC, Hirose K, Lipsett PA. Multifaceted longitudinal study of surgical resident education, quality of life, and patient care before and after July 2011. J Surg Educ. 2013;70(6):76976.

8. Ridic G, Gleason S, Ridic O. Comparisons of health care sys- 
tems in the United States, Germany and Canada. Mater Sociomed.

2012;24(2):112-20.

9. Vogt KN, Allen L, Murphy PB, van Heest R, Saleh F, Widder S, et al. Patterns of complex emergency general surgery in Canada. Can J Surg. 2020;63(5):E435-e41.

10. Charest-Morin R, Flexman AM, Bond M, Ailon T, Dea N, Dvorak $M$, et al. 'After-hours' non-elective spine surgery is associated with increased perioperative adverse events in a quaternary center. European Spine Journal. 2019;28(4):817-28.

11. Dodson TF, Webb AL. Why do residents leave general surgery? The hidden problem in today's programs. Curr Surg. 2005;62(1):128-31.

12. Lee J. Can an Emergency Surgery Scheduling Software Improves Residents' Time Management and Quality of life? [master's thesis]: McGill University; 2019. 